\title{
The Design of Degree Distribution for Distributed Fountain Codes in Wireless Sensor Networks
}

\author{
Jing Yue ${ }^{\dagger}$, Zihuai Lin ${ }^{\dagger}$, Branka Vucetic ${ }^{\dagger}$, and Pei Xiao ${ }^{\ddagger}$ \\ ${ }^{\dagger}$ School of Electrical and Information Engineering, The University of Sydney, Sydney, NSW 2006, Australia \\ ${ }^{\ddagger}$ Department of Electronic Engineering, The University of Surrey, United Kingdom \\ e-mail: \{jing.yue,zihuai.lin,branka.vucetic\}@ sydney.edu.au, p.xiao@surrey.ac.uk
}

\begin{abstract}
In this paper, we first analyse bit error rate (BER) bounds of the distributed network coding (DNC) scheme based on the Luby-transform (LT) codes, which is a class of fountain codes, for wireless sensor networks (WSNs). Then we investigate the effect from two parameters of the degree distributions, i.e., the degree value and the proportion of odd degree, to the performance of the LT-based DNC scheme. Based on the analysis and investigation results, a degree distribution design criteria is proposed for the DNC scheme based on fountain codes over Rayleigh fading channels. We compare the performance of the DNC scheme based on fountain codes using degree distributions designed in this paper with other schemes given in the literature. The comparison results show that the degree distributions designed by using the proposed criteria have better performance.
\end{abstract}

\section{INTRODUCTION}

Wireless sensor networks (WSNs), where a large number of sensor nodes communicate with their common destination node with the help of a group of other sensor nodes, have been attracted a wide attention from both academia and industry recently. By cooperating with other sensor nodes, the performance of the entire network can be improved. An efficient coding scheme to improve the transmission reliability for the cooperative communication networks is distributed coding [1] [3]. An important distributed coding scheme for WSNs, namely, adaptive network coded cooperation (ANCC) [1], has been proposed to simplify the network code design problem to the problem of the design of a code on graph [4]. Based on the basic idea of ANCC, extrinsic information transfer (EXIT) charts are used in the design of irregular low-density generator matrix (LDGM) codes [4] for WSNs over Rayleigh fading channels in [2].

In the aforementioned works, the source nodes are assumed to be capable of communicating with their common destination node directly. However, in some WSN applications, the direct links between the source nodes and the destination node are not available all the time. The data transmission from the source nodes to the destination node appears in a multi-hop fashion. For these applications, a distributed network coding (DNC) scheme based on the Luby-transform (LT) [5] codes, which is a class of fountain codes, has been proposed in [3]. Fountain codes, which is also referred to as rateless codes, is capable of providing reliable transmission by producing a potentially infinite length of codeword. Due to the special property of the fountain codes, the DNC scheme based on fountain codes can be used for applications where direct links are not available between the source nodes and their common destination node.

The design of degree distribution plays a pivotal role in the achievable performance of a fountain code. Let us take the LT codes as an example, the initial degree distribution for the LT codes, namely the Ideal Soliton distribution (ISD) [5], is optimal in theory but inapplicable in practice. Then Robust Soliton distribution (RSD) is developed. The performance of the RSD is significantly better than that of the ISD. Over the years a lot of work has been done on analysis and design of degree distribution for the LT codes [6]-[9].

In this paper, we divide the analytical bit error rate (BER) bounds of the LT-based DNC scheme into two parts and analyse each part separately. From the analysis result we find that it is the parameters of the degree distributions, i.e., the degree value and the proportion of odd degree, affect the performance of the LT-based DNC scheme. The effect from these parameters on the system performance are summarised. Based on the analysis and investigation results, we propose a degree distribution design criteria for the DNC scheme based on fountain codes over Rayleigh fading channels. Finally, we compare our degree distributions with the existing ones in the literature. The results show that by using our degree distribution design criteria, better performance can be achieved. Also, the upper and lower bounds become tighter when our proposed degree distribution design criteria is employed.

\section{SySTEM MODEL}

We consider that there are $M$ sensor nodes and a common destination node in a WSN. Among the $M$ sensor nodes, $\mathrm{K}$ of them have collected data to transmit. Thus, these $K$ sensor nodes, where $K<M$, act as source nodes in the WSN. The rest $N=M-K$ sensor nodes, have no data to transmit and act as relay nodes.

Let $S_{i}, R_{j}$ and $D$ represent the $i$ th source node, the $j$ th relay node, and the destination node, respectively, where $i \in\{1,2, \ldots, K\}$ and $j \in\{1,2, \ldots, N\}$. We assume that all the source nodes in the considered WSN are not capable of communicating with their common destination node directly. It means that in our system model, there is no direct links between the source nodes and the destination node, but there exist direct links between the source and the relay nodes, and 
between the relay nodes and the destination node. The network graph we described above is shown in Fig. 1.

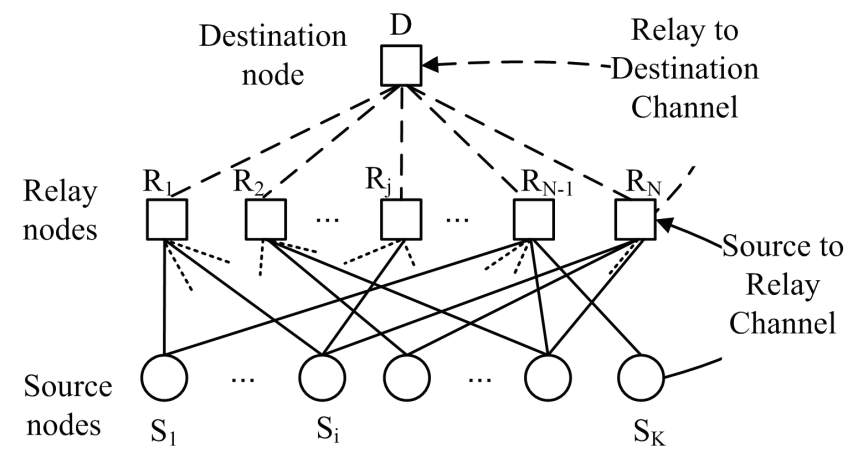

Fig. 1. A network graph used to illustrate the system model.

All the data transmission in the investigated system are in the form of data packets. The data delivery from the source nodes to the destination node is carried out in three phases:

Broadcast phase: First, a header and a cyclic redundance check (CRC) code are added to each source node's information data sequence to form a data packet. The index of the source node is contained in its data packet's header. Then all the source nodes broadcast their data packets to the relay nodes. A certain MAC layer protocol, e.g., Time-Division Multiple Access (TDMA) or Carrier Sense Multiple Access (CSMA), is assumed to be employed. This ensures that the source node transmissions do not cause interference to each other. Note that all the information data sequences of the source nodes have the same length $\epsilon \times 1$ and are assumed to be independent identically distributed (i.i.d).

Relay phase: Each relay node listens to the transmission from the source nodes, decodes the received data packets, and then puts the correctly decoded data together with the indices of the source nodes into its buffer. Then each relay node selects a fixed number $d$ of correctly decoded data from its buffer to perform network coding by using linear combination in the field of $\mathrm{GF}(2)$. The fixed number $d$ is selected corresponding to the degree distribution $\mu(x)=\sum_{d} \mu_{d} x^{d}$. After that, a header and a CRC code are added to each network-coded data to form a network-coded data packet. The connection information, including the indices of the relay node and the source nodes which have been selected by the relay node to form the network-coded data, are contained in the header of each network-coded data packet. Finally, the relay nodes send their network-coded data packets to the destination node.

Data recovery phase: At the destination node, the network topology can be mapped to a code on graph according to the connection information contained in the headers of the received data packets. After receiving a sufficient number, say $Q$, of data packets from the relay nodes, the destination node begins to decode. $Q=\eta K$, where $\eta$ is the expanding coefficient.

The two processes, i.e., the relay phase and the data recovery phase, run on alternately until the destination node can recover all the information successfully.

\section{Analysis of the Analytical Bounds of the LT-BASED DNC SCHEME}

The channels between the source and the relay nodes, and between the relay nodes and the destination node are assumed to be quasi-static Rayleigh fading channels. Let $\hbar_{j, i}^{R S}$ and $\hbar_{j}^{D R}$ represent the fading coefficient for the link between the $i$ th source node $S_{i}$ and the $j$ th relay node $R_{j}$, and between $R_{j}$ and the destination node $D$, respectively. The fading coefficients $\hbar_{j, i}^{R S}$ and $\hbar_{j}^{D R}$ remain constant over the length of one data packet, but change independently from packet to packet. These fading coefficients have zero mean and unit variance [10], i.e., $E\left\{\left|\hbar_{j, i}^{R S}\right|^{2}\right\}=1$ and $E\left\{\left|\hbar_{j}^{D R}\right|^{2}\right\}=1$. Here $E\{\cdot\}$ denotes the expectation.

The information data sequences from all the source nodes are arranged into a matrix $\boldsymbol{\Lambda}=\left[\mathbf{m}_{1}, \mathbf{m}_{2}, \cdots, \mathbf{m}_{K}\right] \in$ $\operatorname{GF}(2)^{\epsilon \times K}$. The $Q$ output network-coded data from the relay nodes can be represented by $\mathbf{V}=\mathbf{\Lambda} \mathbf{G}$, where $\mathbf{V} \in \mathrm{GF}(2)^{\epsilon \times Q}$. $\mathbf{G}$ is a randomly generated $K \times Q$ binary matrix formed at the relay nodes, which is associated with the fountain code. The $j$ th column of $\mathbf{V}$ is the transmitted data packet from the $j$ th relay node. Since the binary information data sequences of the source nodes are assumed to be i.i.d, we only consider an arbitrary row of $\boldsymbol{\Lambda}$ in the analysis and denote it by $\mathbf{m}$. Let $\mathbf{v}$ be the corresponding row of $\mathbf{V}$ when $\mathbf{m}$ is chosen as the input, i.e., $\mathbf{v}=\mathbf{m G}$.

The $(i, j)$ th element of $\mathbf{G}$, which is denoted by $G(i, j)$, is equal to one if the $i$ th source node's data is used to generate the output data at the $j$ th relay node, otherwise it is zero. Let $P_{1}$ and $P_{0}$ represent the probability that $G(i, j)$ is set to be one and zero, respectively. Note that if $P_{1}>\frac{1}{2}$, as the density of one of the generator matrix $\mathbf{G}$ increases, the complexity of encoding and decoding of the codes also increase. Thus, we only consider the case when $P_{1} \leq \frac{1}{2}$. Since the applied codes are linear, their performance is independent of the transmitted information data sequence. Thus, we can assume that the all zero sequence is transmitted, i.e., $\mathbf{m}=\mathbf{0}$. The error information sequence is denoted by $\mathbf{e}$.

Due to the fact that the fading coefficients remain constant over the length of one data packet but change independently among different packets, the channel state information (CSI) is known at the destination node. All the date received at the destination node form a data matrix. The data matrix is decoded line by line. Thus, the decoding process at the destination node can be seen as over fast Rayleigh fading channels but with perfect CSI.

\section{A. Analytical Bounds}

The upper bound of the LT-based DNC scheme has been given in [3]. Also, it has been shown in [3] that the simulation results agree with the analytical upper bound especially when the expanding coefficient $\eta$ is large. The upper bound of the LT-based DNC scheme can be summarised as:

For the LT-based DNC scheme with parameters $\mu(x), K$, $Q$ and expanding coefficient $\eta$, its bit error performance under maximum-likelihood (ML) decoding over Rayleigh fading 
channels can be upper bounded by $\xi_{U}<\max \left\{1, \xi_{U}^{L T}\right\}$, where $\xi_{U}^{L T}$ is given by

$$
\begin{aligned}
\xi_{U}^{L T}= & \sum_{k=1}^{K} \frac{k}{K}\left(\begin{array}{c}
K \\
k
\end{array}\right)\left\{\left[\sum_{w(\mathbf{c})} \mu_{w(\mathbf{c}) \varsigma_{k}}(w(\mathbf{c}))\right]^{Q}\right. \\
& +\sum_{\kappa=1}^{Q} \frac{1}{\pi}\left(\begin{array}{c}
Q \\
\kappa
\end{array}\right) \beta_{k}^{\kappa}\left(1-\beta_{k}\right)^{Q-\kappa} \\
& \left.\cdot \int_{0}^{\pi / 2}\left(\frac{1}{1+\frac{E_{s}^{\kappa}}{N_{0} \sin ^{2}(\theta)}}\right)^{\kappa} d \theta\right\}
\end{aligned}
$$

where

$$
\begin{aligned}
& \varsigma_{k}(w(\mathbf{c}))=\frac{\sum_{\alpha=\text { even }, \alpha \leq k}\left(\begin{array}{l}
k \\
\alpha
\end{array}\right)\left(\begin{array}{c}
K-k \\
w(\mathbf{c})-\alpha
\end{array}\right)}{\left(\begin{array}{c}
K \\
w(\mathbf{c})
\end{array}\right)}, \\
& \beta_{k}=\sum_{w(\mathbf{c})} \mu_{w(\mathbf{c})}\left(1-\varsigma_{k}(w(\mathbf{c}))\right)
\end{aligned}
$$

and $Q=\eta K, w(\cdot)$ denotes the Hamming weight, c represents an arbitrary column of the matrix $\mathbf{G}, \mu_{w(\mathbf{c})}$ is the probability that the column vector c has weight $w(\mathbf{c})$.

The lower bound on the bit error probability for the LTbased DNC scheme under ML decoding over Rayleigh fading channels is given by the probability that only one symbol in the decoded sequence is not recovered correctly [6].

In the following, we focus on analysing the performance of the LT-based DNC scheme from the analytical bounds.

\section{B. Analysis of the Analytical Bounds}

We divide the upper bound in (1) into two parts, and analyse each part of the upper bound separately.

The first part of the upper bound can be written as

$$
\sum_{k=1}^{K} \frac{k}{K}\left(\begin{array}{c}
K \\
k
\end{array}\right)\left[\sum_{w(\mathbf{c})} \mu_{w(\mathbf{c})} \frac{\sum_{\alpha=\text { even }, \alpha \leq k}\left(\begin{array}{c}
k \\
\alpha
\end{array}\right)\left(\begin{array}{c}
K-k \\
w(\mathbf{c})-\alpha
\end{array}\right)}{\left(\begin{array}{c}
K \\
w(\mathbf{c})
\end{array}\right)}\right]^{Q} .
$$

Since (2) has the same form as the upper bound given in [11], which is derived for the LT codes over binary erasure channels (BECs), we can use the analysis results in [11] directly. The conclusion given in [11] is that if keep the other parameters unchanged, as $P_{1}$ increases, the value of (2) decreases. When $P_{1}=\frac{1}{2}$, (2) reaches the minimum value.

We next check whether this conclusion for the first part of (1) also holds for its second part. Let $B_{1}(f)$ and $B_{2}(f)$ represent the two parts of (1), respectively, then $\xi_{U}^{L T}$ in (1) can be written as $\xi_{U}^{L T}=B_{1}(f)+B_{2}(f)$. Let

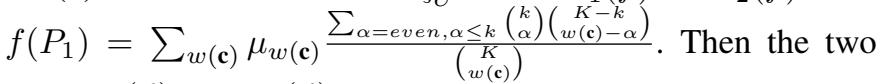
parts $B_{1}(f)$ and $B_{2}(f)$ of the upper bound can be represented as follows:

$$
\begin{aligned}
B_{1}(f)= & \sum_{k=1}^{K} \frac{k}{K}\left(\begin{array}{c}
K \\
k
\end{array}\right) f\left(P_{1}\right)^{Q} \\
B_{2}(f)= & \sum_{k=1}^{K} \frac{k}{K}\left(\begin{array}{c}
K \\
k
\end{array}\right) \sum_{\kappa=1}^{Q} \frac{1}{\pi}\left(\begin{array}{l}
Q \\
\kappa
\end{array}\right)\left(1-f\left(P_{1}\right)\right)^{\kappa} \\
& \cdot f\left(P_{1}\right)^{Q-\kappa} \int_{0}^{\pi / 2}\left(\frac{1}{1+\frac{E_{s}^{\kappa}}{N_{0} \sin ^{2}(\theta)}}\right)^{\kappa} d \theta
\end{aligned}
$$

We first analyse the relationship between $P_{1}$ and $f\left(P_{1}\right)$, where $P_{1}=\frac{d_{a v g}}{K}$. Here $d_{a v g}=\sum_{d} \mu_{d} d$ is the average degree of the LT codes. Let $d_{o d d}$ and $d_{\text {even }}$ represent the degree with an odd number and an even number, respectively. As can be seen from Fig. 2, when $k \leq \frac{K}{2}$, the value of $f\left(P_{1}\right)$ is almost the same between the two consecutive degrees. When $k>\frac{K}{2}$, the value of $f\left(P_{1}\right)$ with odd degrees are significant smaller when that with even degrees.

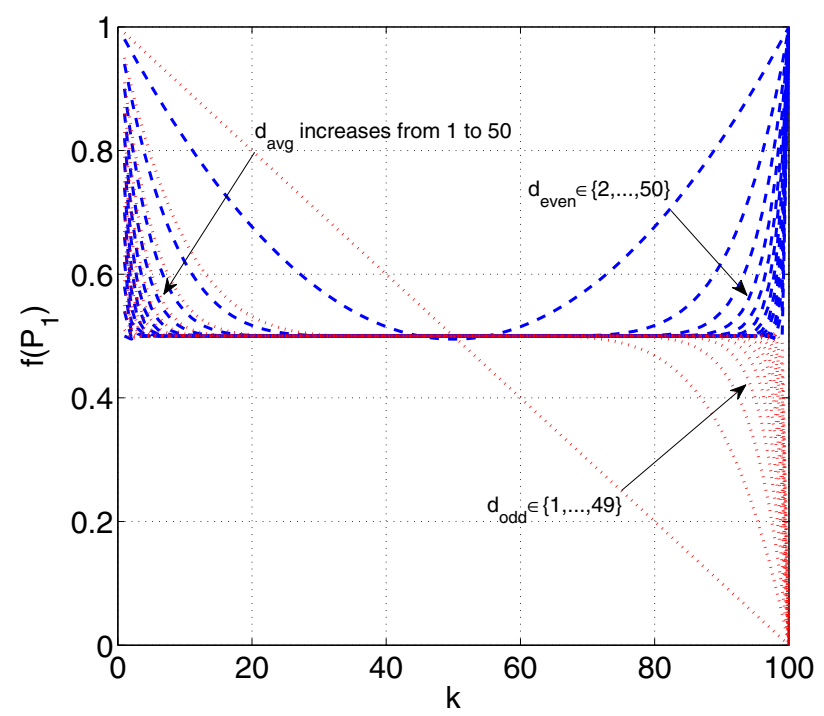

Fig. 2. $f\left(P_{1}\right)$ when the degree is an even or an odd number.

Then let us examine how the value of $B_{2}(f)$ changes with $f\left(P_{1}\right)$. As shown in Fig. 3, the value of $B_{2}(f)$ decreases with the decreasing of $f\left(P_{1}\right)$. When $f\left(P_{1}\right)$ reaches the minimum value, $B_{2}(f)$ reaches its smallest value as well.

Therefore, we can come to the conclusion that if all the degrees in forming a degree distribution with $d_{a v g}=\frac{K}{2}$ (i.e., $P_{1}=\frac{1}{2}$ ) are odd numbers, the upper bound in (1) would reach the lowest one of all its possible positions. Substitute $P_{1}=\frac{1}{2}$ into the two parts of the upper bound, $B_{1}(f)$ and $B_{2}(f)$, respectively, we obtain the the following expression for $\xi_{U}^{L T}$ :

$$
\xi_{U}^{L T}=2^{K(\eta-1)+1}\left[1+\sum_{\kappa=1}^{Q} \frac{1}{\pi}\left(\begin{array}{l}
Q \\
\kappa
\end{array}\right)\right.
$$




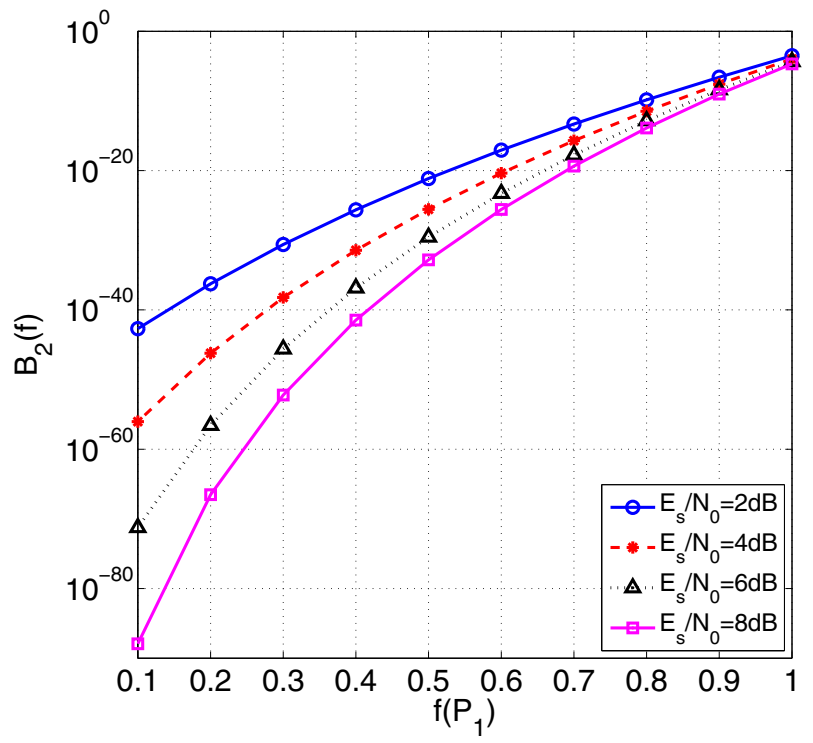

Fig. 3. The second part of the upper bound, $B_{2}(f)$, changes with $f\left(P_{1}\right)$.

$$
\left.\int_{0}^{\pi / 2}\left(\frac{1}{1+\frac{E_{s}^{k}}{N_{0} \sin ^{2}(\theta)}}\right)^{\kappa} d \theta\right] .
$$

With the same derivation for the case, when $P_{1}=\frac{1}{2}$, we obtain the lower bound of the LT-based DNC scheme in the following form:

$$
\begin{aligned}
\xi_{L}^{L T}= & 2^{\eta K}\left[1+\sum_{\kappa=1}^{Q} \frac{1}{\pi}\left(\begin{array}{c}
Q \\
\kappa
\end{array}\right)\right. \\
& \left.\cdot \int_{0}^{\pi / 2}\left(\frac{1}{1+\frac{E_{s}^{\kappa}}{N_{0} \sin ^{2}(\theta)}}\right)^{\kappa} d \theta\right] .
\end{aligned}
$$

The upper and lower bounds given in (3) and (4) are the lowest ones of all their possible positions. Now the question is whether $P_{1}=\frac{1}{2}$ is the only point at which both the upper and lower bounds can reach their lowest positions. We will analyse this problem in the sequel. Also, the relationship between the degree distribution and the performance will be investigated.

\section{The Degree Value and the Proportion of Odd Degree}

When $P_{1}=\frac{1}{2}$ and all the degrees are chosen to be odd numbers, both the upper and lower bounds of the LT-based DNC scheme reach their lowest positions, however, at this point, the gap between the two bounds is the biggest. As $P_{1}$ decreases, the upper and lower bounds asymptotically converge, but the performance becomes worse in the meantime. Thus, we need to find some points at which the LT-based DNC scheme can achieve its optimal performance and meanwhile the upper and lower bounds are asymptotically tight.

We take the case when $K=100$ and $E_{s} / N_{0}=5 \mathrm{~dB}$ as an example to illustrate the effect of the degree value and the proportion of odd degree on the performance. Fig. 4 shows the upper and lower bounds for the LT-based DNC scheme with different average degree, and all of the degrees are with odd numbers. From Fig. 4 we can see that when the average degree is small, e.g., $d_{a v g} \leq 11$, the upper and lower is asymptotically tight as the expanding coefficient $\eta$ grows. Also, it can be seen that as $d_{a v g}$ increases, the position of both the two bounds become lower. After $d_{a v g}=19$, as the average degree increases, the lower bound continues changing to lower positions, while the changes of the upper bound are negligible small.

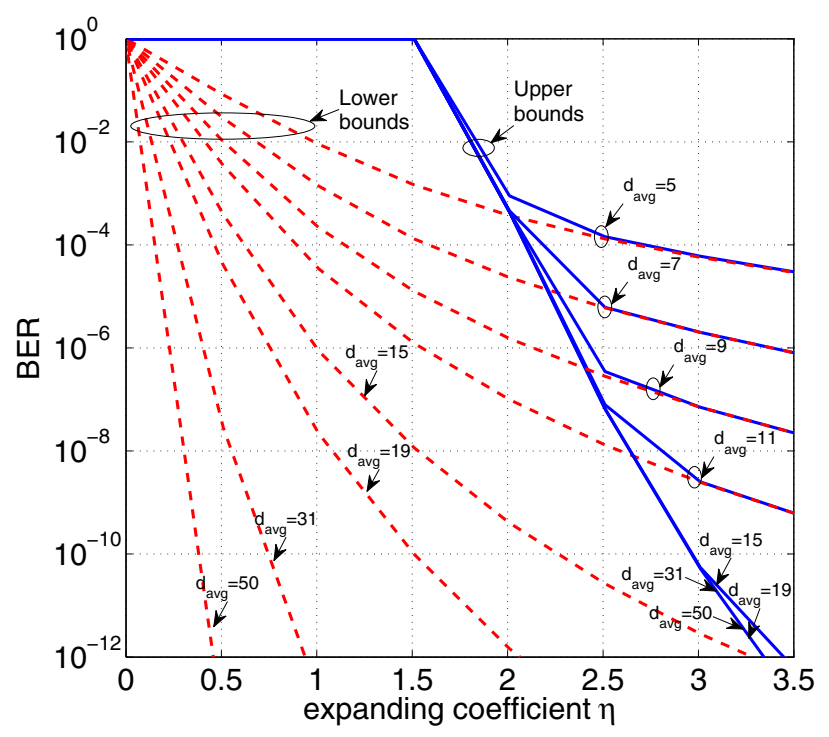

Fig. 4. The upper and lower bounds with different average degree $d_{\text {avg }}$ when $K=100$ and $E_{s} / N_{0}=5 \mathrm{~dB}$.

Fig. 5 shows the upper and lower bounds with different proportion of odd degrees in the degree distributions with average degree $d_{\text {avg }}=19$ when $K=100$ and $E_{s} / N_{0}=5 \mathrm{~dB}$. Let $P_{\text {odd }}=\sum_{d=o d d} \mu_{d}$ represent the proportion of odd degrees in a degree combination. As shown in Fig. 5, as $P_{\text {odd }}$ increases, the upper bound changes to lower positions, while the lower bound keeps unchanged all the time. After $P_{\text {odd }}=0.18$, though the proportion of odd degrees continues increasing, the changes of the upper bound are negligible small.

Consequently, we can come to a conclusion that when $K=100$ and $E_{s} / N_{0}=5 \mathrm{~dB}$, if the degrees are chosen satisfying the two limitations, i.e., $d_{\text {avg }}=19$ and $P_{\text {odd }}=0.18$, simultaneously, the performance of the LT-based DNC scheme would be nearly as good as that for $P_{1}=\frac{1}{2}$.

For different $E_{s} / N_{0}$, the requirements for the degree parameters $d_{\text {avg }}$ and $P_{\text {odd }}$ in order to achieve the near optimal performance are different. Table I shows the average degree $d_{\text {avg }}$ and the proportion of odd degree $P_{\text {odd }}$ required to obtain the near optimal performance at different $E_{s} / N_{0}$ when $K=100$. From Table I we can see that as $E_{s} / N_{0}$ increases, both the average degree and the proportion of odd degree required to achieve near optimal performance increase as well. For example, when $E_{s} / N_{0}=4 \mathrm{~dB}, d_{a v g}=12$ and 
TABLE I

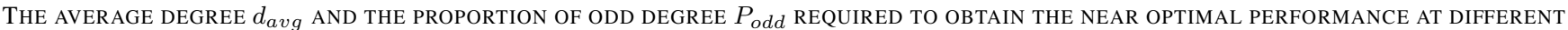
$E_{s} / N_{0}$ WHEN $K=100$.

\begin{tabular}{|l|c|c|c|c|c|c|c|c|c|}
\hline$E_{s} / N_{0}(\mathrm{~dB})$ & 4 & 4.5 & 5 & 5.5 & 6 & 7 & 8 & 9 & 10 \\
\hline$d_{\text {avg }}$ & 12 & 16 & 19 & 20 & 22 & 27 & 29 & 31 & 33 \\
\hline$P_{\text {odd }}$ & 0.13 & 0.16 & 0.18 & 0.21 & 0.23 & 0.28 & 0.3 & 0.33 & 0.33 \\
\hline
\end{tabular}

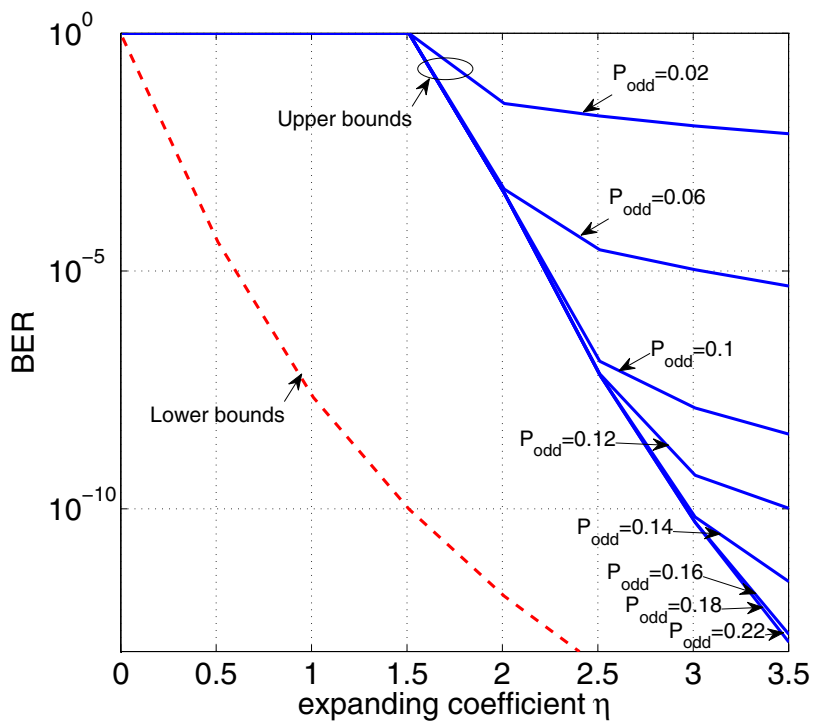

Fig. 5. The upper and lower bounds with different proportion of odd degree $P_{\text {odd }}$ when $K=100$ and $E_{s} / N_{0}=5 \mathrm{~dB}$.

$P_{\text {odd }}=0.13$ are required. When $E_{s} / N_{0}$ increases to $8 \mathrm{~dB}$, then $d_{\text {avg }}=29$ and $P_{\text {odd }}=0.3$ are required.

\section{Design of Degree Distribution}

In this section, we first propose a degree design distribution criteria for the DNC scheme based on fountain codes over Rayleigh fading channels. Then we compare the performance of the degree distributions designed in this paper with that given in the literature.

\section{A. Degree Distribution Design Criteria}

The degree distribution design criteria for the DNC scheme based on fountain codes over Rayleigh fading channels is summarised as:

- First, choose a proper average degree $d_{a v g}$ according to the system parameters, including the total number of source nodes $K$ in a WSN, the system performance requirement, and the channel parameter $E_{s} / N_{0}$.

- Then, choose a proper proportion of odd degrees $P_{\text {odd }}$. To obtain the required performance, the proportion of odd degrees should be chosen equal to or larger than a special value. The special value, which is shown in Table I, is set according to the number of source nodes $K$, the channel parameter $E_{s} / N_{0}$, and the selected average degree $d_{a v g}$.

- Finally, construct a degree distribution according to the chosen parameters $d_{a v g}$ and $P_{o d d}$. After the proportion of odd degrees is satisfied, the rest degree values can be either odd or even numbers. The order of odd and even degrees in the degree distribution does not affect the overall performance.

From the description above we can see that the complexity of constructing degree distributions using the design criteria we proposed in this paper is as low as the method given in [11], and is much lower than that given in [6].

\section{B. Comparison of the Degree Distributions}

We compare the performance of the degree distributions designed by using our proposed criteria with that given in the literature.

The degree distributions in [11] are designed for the BECs, and the degree values can only be chosen from odd numbers. While our degree distribution is designed for Rayleigh fading channels. Both odd and even numbers can be used in the construction of degree distributions. The only thing we have to ensure is that the proportion of odd degrees in the degree distribution is equal to or larger than the selected proportion $P_{\text {odd }}$.

Now let us compare the performance of the degree distributions designed using our proposed criteria with the degree distribution given in [6]:

$$
\begin{aligned}
\mu(x)= & 0.007969 x+0.493570 x^{2}+0.166220 x^{3} \\
& +0.072646 x^{4}+0.082558 x^{5}+0.056058 x^{8} \\
& +0.037229 x^{9}+0.055590 x^{19}+0.025023 x^{65} \\
& +0.003135 x^{66}
\end{aligned}
$$

The average degree $d_{\text {avg }}$ of the degree distribution in 5 is equal to 5.87 , and its proportion of odd degree $P_{\text {odd }}=$ 0.375 . We construct a new degree distribution with an average degree $d_{\text {avg }}=5.87$ using the proposed design criteria in this paper. The generator polynomial of the new designed degree distribution is

$$
\begin{aligned}
\mu(x)= & 0.2 x^{2}+0.35 x^{5}+0.3 x^{7}+0.115 x^{10} \\
& +0.01 x^{12}+0.025 x^{14}
\end{aligned}
$$

Note that the performance of the DNC scheme based on fountain codes using different degree distributions is the same if degree parameters $d_{a v g}$ and $P_{o d d}$ of these degree distributions are the same. The degree distribution in (6) is randomly selected from the degree distributions which have $d_{\text {avg }}=5.87$ and $P_{\text {odd }}=0.65$. In order to compare the performance, we also construct degree distributions with $d_{a v g}=9$ and $P_{\text {odd }}=0.65$, and randomly select one from these constructed 
degree distributions. The randomly selected degree distribution can be represented as

$\mu(x)=0.095 x^{2}+0.2 x^{7}+0.3 x^{9}+0.15 x^{11}+0.255 x^{12}$.

Figs. 6(a) and 6(b) show the analytical bounds of the constructed fountain codes ensemble using the degree distributions we designed in (6) and (7), and the degree distribution (5) given in [6]. The other parameters are $K=100, E_{s} / N_{0}=5$ and $7 \mathrm{~dB}$, respectively. The expanding coefficient $\eta$ changes from 1.0 to 3.5. From Fig. 6(a) we can see that when $E_{s} / N_{0}=5 \mathrm{~dB}$ and $d_{a v g}=5.87$, the degree distribution we designed has better performance than the one given in [6]. It means that by changing the proportion of odd degree in the degree distribution, better performance can be achieved. We can also see from the figure that the performance of the degree distribution with average degree $d_{\text {avg }}=9$ is much better than that with $d_{\text {avg }}=5.87$. It means that a larger average degree brings better performance. Also, it can be seen in Fig. 6(a) that the upper and lower bounds constructed by using our proposed degree distributions are much tighter than the bounds constructed by using the degree distribution given in [6].

As shown in Fig. 6(b), the comparison result remains the same when $E_{s} / N_{0}$ increases to be a larger number, i.e., $E_{s} / N_{0}=7 \mathrm{~dB}$. In summary, regardless of the value of $E_{s} / N_{0}$ and the expanding coefficient $\eta$, the DNC scheme based on fountain codes using the degree distributions designed by our proposed design criteria has better performance than that given in [6].

\section{CONClusion}

In this work, we analysed the analytical upper and lower bounds for the LT-based DNC scheme under ML decoding over Rayleigh fading channels. We summarised the effect from the degree value and the proportion of odd degree to the performance of the LT-based DNC scheme. Degree distribution design criteria was proposed for the DNC scheme based on fountain codes over Rayleigh fading channels. By comparison, we showed that our degree distribution design yields better performance than other existing schemes in the literature.

\section{REFERENCES}

[1] X. Bao and J. Li, "Adaptive network coded cooperation (ANCC) for wireless relay networks: matching code-on-graph with network-ongraph," IEEE Trans. Wireless Commun., vol. 7, no. 2, pp. 574-583, Feb. 2008.

[2] K. Pang, Z. Lin, Y. Li and B. Vucetic, "Design of distributed networkchannel codes for wireless sensor networks," in Proc. of IEEE ICC'11, Kyoto, Japan, Jun. 2011, pp. 1-5.

[3] K. Pang, Z. Lin, B. F. Uchôa-Filho and B. Vucetic, "Distributed network coding for wireless sensor networks based on rateless LT codes," IEEE Wireless Commum. Lett., vol. 1, no. 6, pp. 561-564, Dec. 2012.

[4] J. Garcia-Frias and W. Zhong, "Approaching Shannon performance by iterative decoding of linear codes with low-density generator matrix," IEEE Commum. Lett., vol. 7, no. 6, pp. 266-268, Jun. 2003.

[5] M. Luby, "LT codes," in Proc. of $43^{\text {rd }}$ Annu. IEEE Symp. FOCS., Vancouver, BC, Canada, Oct. 2002, pp. 271-280.

[6] A. Shokrollahi, "Raptor codes," IEEE Trans. Inform. Theory, vol. 52, no. 6, pp. 2551-2567, Jun. 2006.

[7] H. Zhu, G. Zhang and G. Li, "A novel degree distribution algorithm of LT codes," in Proc. of IEEE ICCT'08, Nov. 2008.

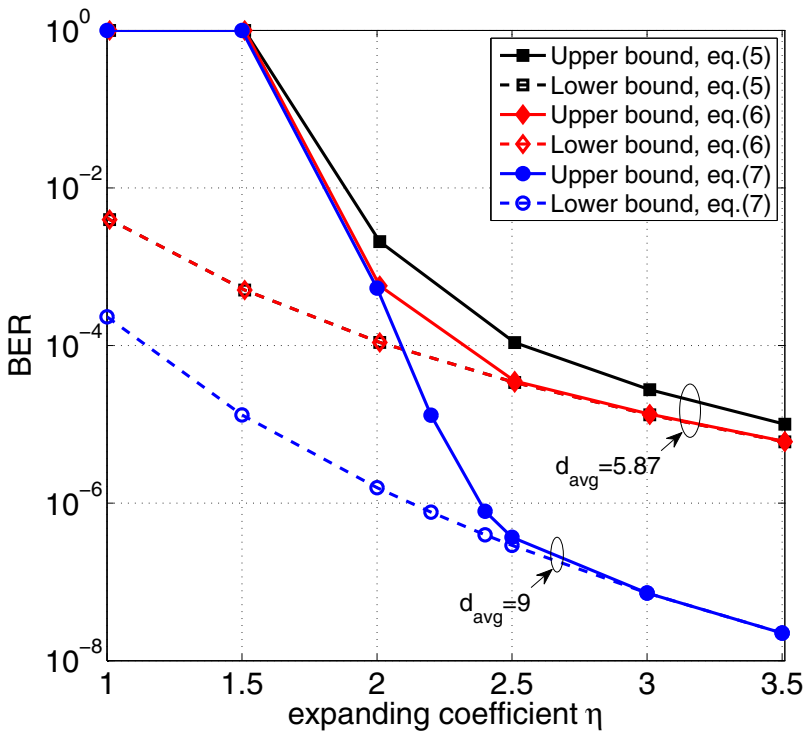

(a) $K=100, E_{s} / N_{0}=5 \mathrm{~dB}$.

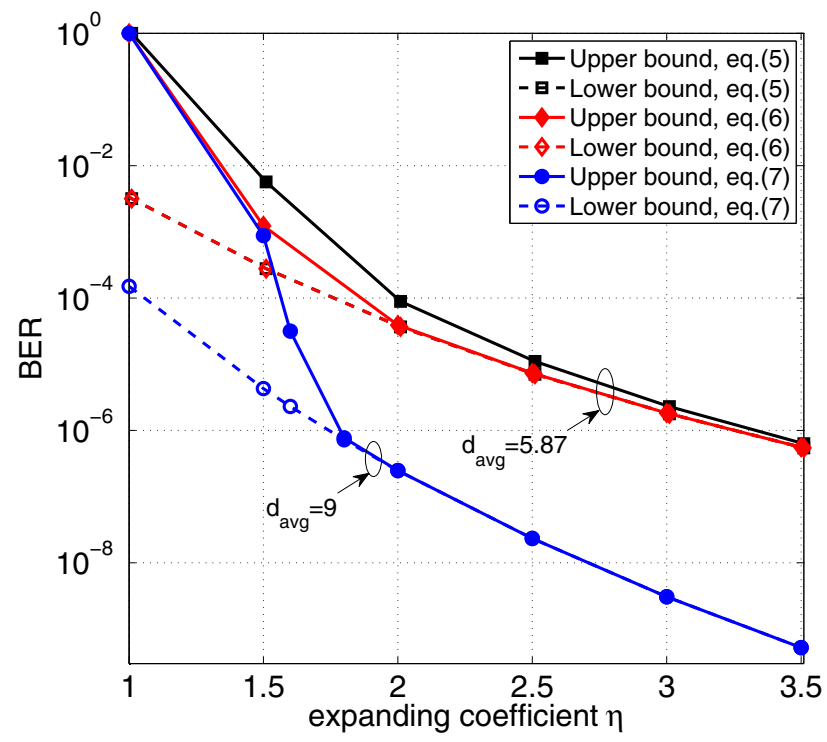

(b) $K=100, E_{s} / N_{0}=7 \mathrm{~dB}$.

Fig. 6. The performance comparison of the degree distributions designed in this paper with that given in the literature when $K=100, E_{s} / N_{0}=5$ and $7 \mathrm{~dB}$, respectively.

[8] G. Maatouk and A. Shokrollahi, "Analysis of the second moment of the LT decoder," IEEE Trans. Inform. Theory, vol. 58, no. 5, pp. 2558-2569, May 2012.

[9] J. Sørensen and P. Popovski, "Design and analysis of LT codes with decreasing ripple size," IEEE Trans. Communications, vol. 60, no. 11, pp. 3191-3197, Nov. 2012.

[10] Y. Xi, A. Burr, J. Wei and D. Grace, "A general upper bound to evaluate packet error rate over quasi-static fading channels," IEEE Trans. Communications, vol. 10, no. 5, pp. 1373-1377, May. 2011.

[11] B. Schotsch, H. Schepker and P. Vary, "The performance of short random linear fountain codes under maximum likelihood decoding," in Proc. of IEEE ICC'11, Kyoto, Japan, Jun. 2011, pp. 1-5. 